\title{
M-BAND PERFECT-RECONSTRUCTION LINEAR-PHASE FILTER BANKS
}

\author{
X. M. Xie, S. C. Chan and T. I. Yuk \\ Department of Electrical and Electronic Engineering \\ University of Hong Kong. Pokfulam Road, Hong Kong. \\ \{xmxie, scchan, tiyuk\}@eee.hku.hk
}

\begin{abstract}
This paper studies the design of $M$-channel perfectreconstruction (PR) linear-phase (LP) filter banks (FBs) with $M=2^{K}$ using a tree-structured FB. It is based on a observation of Fliege [1], the length of the analysis filters is decreased by a factor of two when the depth of the tree is increased by one, while its transition bandwidth is increased by the same factor. A lattice-based 2-channel LP FB is chosen because the frequency responses of the lowpass and highpass analysis (synthesis) filters can be designed to be closely symmetric to the other around $\pi / 2$. By properly selecting the filter length, transition bandwidth. and stopband attenuation of the 2-channel PR LP FBs at each level of the tree structure, it is possible to design uniform PR LP FB with excellent frequency characteristic and much lower system delay.
\end{abstract}

\section{INTRODUCTION}

Perfect-reconstruction (PR) linear-phase (LP) filter banks (FBs) are used in a wide range of applications, such as data compression, communications, and image and speech coding. Fig. 1 shows the block diagram of a critically decimated $M$-band uniform FB. The input signal is first decomposed by $M$ analysis filters $H_{i}(z)$. The outputs are then decimated by a factor of $M$ to form $M$ subband signals. In the synthesis bank, the subband signals are upsampled by a factor of $M$ before passing through the synthesis filters $F_{i}(z)$ to reconstruct the processed signal. The theory and design of PR FB have been widely studied in the literature [2]. One efficient structure is the cosine-modulated (CMFB), where the analysis filters (synthesis filters) are obtained by cosine modulation of a prototype filter. Due to the cosine modulation, the implementation and design complexities of CMFB are very low compared with a general PR FBs. Unfortunately, the classical CMFB proposed in [3] does not have LP analysis and synthesis filters, which is desirable in some applications. More recently, a new class CMFB using a different cosine and sine modulations are proposed [4]. Although the analysis and synthesis filters are LP, its frequency support is considerably different from that of uniform FB and there is considerable overlap between the passband of the low frequency analysis filters. Another popular class of LP $M$-channel uniform FBs is the linear-phase paraunitary filter bank (LPPUFB) [5], where the LP FB is parameterized as a cascade of delays and unitary matrices, which can further be parameterized as a series of planar rotations. The design of LPPUFB can be very involved because of the large number of design parameters and the highly nonlinear dependency of the frequency response on the rotation parameters [5-8]. This usually limits the stopband attenuation of the FB. Another commonly used method to construct PR FB is to cascade sets of PR FBs with smaller number of channels in a tree structure [9]. For example, an 8-channel PR FB can be obtained by cascading sets of 2-channel PR FBs in a tree structure with 3 levels as shown in Fig. 2. The output from the previous level is further decomposed using the analysis filters in that level into two more channeis. In general, all the 2-channel PR FBs can differ from each other and they can be either linear- or nonlinear-phase. In wavelet transform and most tree-structured FBs considered in the literature, the same set of PR FB is used throughout the tree structure. Two significant drawbacks of this structure, from the viewpoint of designing a uniform $\mathrm{FB}$, are the high system delay and the asymmetric transition band of the analysis filters. The latter usually results in a higher filter order to satisfy a given stopband attenuation and transition bandwidth, which further increases the total system delay. This is illustrated in Fig. 3 using a 2-channel PR FB with filter length $N=128$. It can be seen that transition bandwidth are unequal and the system delay rapidly increases to 889 samples. In [1]. Fliege has shown that the system delay of a tree-structured FB can be drastically reduced by having non-identical analysis filters in each level of the tree. More precisely, the length of the filters should decrease by a factor of two when going from one level to the other, while their transition bandwidth should increase by the same factor. In this paper, we further study this novel idea in the design of $M$-channel LP uniform FB using the lattice-based two-channel LP FB proposed in [10]. The main reason in choosing the latter is that the frequency responses of the lowpass and highpass analysis (synthesis) filters can be designed to be closely symmetric to the other around $\pi / 2$. By properly selecting the filter length. transition bandwidth, and stopband attenuation of the 2-channel PR LP FBs at each level of the tree structure, it is possible to design uniform LP FB with excellent frequency characteristic and much lower system delay. For example, a uniform 8-channel PR LP FB with the same worse-case transition bandwidth requirement and stopband attenuation as the previous one $(N=128)$ can be achieved with the new structure with a much lower implementation complexity and system delay of 377 samples (Fig. 4). The savings also increase linearly with the depth of the tree structure. The resulting FB, therefore, serves as useful alternative to the LPPUFB for designing LP FB with $N$ a powers of two number and more generally $M$ a composite number. Though the design of the component LPPUFBs in the latter case will become more complicated than the 2-channel LP FB in the former, it is still much simpler than designing directly an $M$ channel LPPUFB. The rest of the paper is organized as follows: Section II is devoted to the proposed tree-structured PR LP FB. The design procedure and some design examples are given in Section III. Conclusions are drawn in Section IV.

\section{TREE-STRUCTURED PR LP FBS}

First of all, let's consider an 8-channel tree-structured uniform FB constructed by cascading 2-channel PR FBs as shown in Fig. 2. $H_{0}^{(k)}(z)$ and $H_{i}^{(k)}(z)$ are respectively the lowpass and highpass analysis filters of the 2-channel PR FB at the $k$-th level of the tree structure, where $1 \leq k \leq K$, and $K$ is the total number of levels in the tree. In the synthesis bank, the subband signals are recombined successively, two at a time, by a set of synthesis filters $F_{i}^{(k)}(z)$. From the noble identity, we know that $H(z)$ followed by a decimator with a ratio of two is equivalent to $H\left(z^{2}\right)$ preceding the decimator. Therefore, the tree-structured FB can be redrawn as an 8-channel uniform FB shown in Fig. 1 by moving the analysis filters to the right hand side of the tree structure, leading to $M$ analysis filters $H_{n}(z), m=1, \ldots, M$. For convenience, let's treat the index " $\vec{i}$ " in $H_{i}^{(k)}(z)$ as the $k$-th 
digits in a weighted binary representation and denote it by $b_{k}$. The equivalent transfer function obtained by passing the signal though the branch $H_{b_{1}}^{(1)}(z), H_{b_{z}}^{(2)}(z), \ldots, H_{b_{K}}^{(K)}(z)$ can then be labeled as $H_{n}(z)$, where $m=b_{1}+2 b_{2}+\cdots+2^{K-1} b_{K}=\left(b_{1}, \ldots . b_{K}\right)_{2}$. The resulting analysis filters $H_{m}(z)$ and synthesis filters $F_{m}(z)$ $(0 \leq m \leq M-1)$ can then be written as

$$
\begin{aligned}
& H_{m}(z)=H_{b_{1}}^{(1)}\left(z^{2^{0}}\right) H_{b_{z}}^{(2)}\left(z^{2^{2}}\right) \ldots H_{b_{k}}^{(k)}\left(z^{2^{b-1}}\right) \ldots H_{b_{K}}^{(K)}\left(z^{2^{K-1}}\right) \\
& F_{m l}(z)=F_{b_{1}}^{(1)}\left(z^{2^{0}}\right) F_{b_{2}}^{(2)}\left(z^{2^{1}}\right) \ldots F_{b_{k}}^{(k)}\left(z^{z^{(-1}}\right) \ldots F_{b_{K}}^{(K)}\left(z^{2^{K-1}}\right)
\end{aligned}
$$

It is clear that the whole system is PR if the 2-channel FBs in each level are PR. Further, if $H_{i}^{(k)}(z)$ and $F_{i}^{(k)}(z)$ are LP, then so are the filters $H_{m}(z)$ and $F_{m}(z)$.

As mentioned earlier. if the same set of FB is employed at all levels in the tree-structured FB, then the frequency responses of the overall analysis filters are not identical due to the upsampling of $z$ in moving the analysis filters to left of the decimators, Fig. 3 . As a result, higher implementation complexity is required to achieve a given transition bandwidth and stopband attenuation. This also significantly increases the system delay of the FB. For example, if the lattice-based PR LP FB in [10] is used as the 2-channel FB, the system delay $D$ of the 3-level tree-structured FB is given by

$$
D=N^{(1)}+2\left(N^{(2)}+2\left(N^{(3)}-1\right)-1\right)-1 .
$$

where $N^{(k)}$ is the length of FB in the $k$-th level. If we set $N^{(1)}=N^{(2)}=N^{(3)}=128$, then the resulting tree-structured FB, as shown in Fig. 3, will have a system delay of 889 samples. It can be seen that 2-channel PR FBs at different levels of the tree structure contribute differently to the total system delay. Each component is linearly proportional to the length of the FB used and its scalar constant grows exponentially with the depth or level of the FB in the FB tree. To reduce the total system delay, it is therefore advantageous to reduce the length of the FB when the level increases.

From the design of 1-D FIR filter using the Kaiser window method, we know that the length of the filter $N$, stopband attenuation $A$, and transition bandwidth $\Delta \omega$ are related by the following formula

$$
N=\frac{A-8}{2.285 \cdot \Delta \omega} .
$$

For a given stopband attenuation and passband ripple, the filter length is inversely proportional to the transition bandwidth. From (4), it can be seen that the worse case stopband attenuation of $H_{n i}(z)$ is equal to the worse case stopband attenuation of its factors $H_{b_{1}}^{(1)}\left(z^{2^{0}}\right), \ldots, H_{b_{K}}^{(K)}\left(z^{2^{K-1}}\right)$ and its transition bandwidth will depend on those of $H_{b_{1}}^{(1)}\left(z^{2^{0}}\right), \ldots$, and $H_{b_{K}}^{(K)}\left(z^{2^{K-1}}\right)$. Due to the upsampling by a factor of $2^{k-1}$, the transition bandwidth of $H_{b_{k}}^{(i)}\left(z^{z^{k-1}}\right)$ will be $2^{k-1}$ times narrower than that of $H_{b_{k}}^{(i)}(z)$. Thus, to achieve a uniform transition bandwidth, the length of 2channel PR FB should be reduced by a factor of 2 when we go from one level to the other. In other words,

$$
\begin{aligned}
& N^{(1)}=2 \times N^{(2)}=4 \times N^{(3)} \cdots=2^{K-1} \times N^{(K)} \\
& \Delta^{(1)}=\Delta^{(2)} / 2=\Delta^{(3)} / 4=\cdots=\Delta^{(K)} / 2^{K-1} .
\end{aligned}
$$

The system delay $D$ (for $K=3$ ) is now reduced to $D=3 N^{(1)}-7$, which grows only linearly with the depth of the tree. The arithmetic complexity. in terms of the numbers of multiplications and additions per unit time (MPUs and APUs), also reduced from
99 MPUs and 291 APUs to 59 MPUs and 171 APUs. This novel technique has been mentioned in [1] but unfortunately the selection of the 2-channel PR FB and detail design examples are missing. In this work, we shall show that this approach can be used to obtain $M$-channel LP PR uniform FB ( $M=2^{K}, m$ a positive integer) with very good frequency characteristic, using the lattice-based 2-channel LP PR FB proposed in [10]. Although the efficient structure by Phoog et al [11] can also be used in a similar manner, which is very attractive because of their low design and implementation complexities [12], the frequency responses of its associated lowpass and highpass filters are not quite symmetric to each other. Therefore, the frequency characteristic at the transition band edges will start to degrade when we they are cascaded to form a tree structure with large number of channels. If one is comfortable with nonlinear-phase FIR filters (i.e. only passband linear-phase), then the $\mathrm{CQF}[9]$ and the general low-delay 2-channel FB [13] can also be employed. The latter will further reduce the total system delay of the FB. Interested readers are referred to [14] for more details regarding their design and factorization. We now consider the design of the 2-channel lattice-based LP FB and some design examples.

\section{DESIGN PROCEDURE AND EXAMIES}

\section{A. DESIGN PROCEDURE} given by

For a 2-channel critically decimated FB, the PR condition is

$$
H_{0}^{(k)}(-z) H_{1}^{(k)}(z)-H_{0}^{(k)}(z) H_{1}^{k}(-z)=\beta \cdot z^{-d},
$$

where $d$ is a positive integer and $\beta$ is a nonzero constant. The synthesis filters are given by $F_{0}^{(k)}(z)=H_{1}^{(k)}(-z)$ and $F_{1}^{(k)}(z)=-H_{0}^{(k)}(-z)$. For our LP FB, $H_{0}^{(k)}(z)$ and $H_{1}^{(k)}(z)$ are chosen respectively to be symmetric and antisymmetric having the same filter length, which is an even number. Instead of optimizing the lattice coefficients, which involves highly nonlinear objective function, the coefficients of filters $H_{0}^{(k)}(z)$ and $H_{1}^{(k)}(z)$ are obtained by solving the following constrained optimization

$$
\begin{aligned}
\min _{k} \phi^{(k)} & =\sigma \int_{0}^{\pi-\omega_{s}^{(k)}}\left(1-\left|H_{0}^{(k)}\left(e^{j \omega}\right)\right|\right)^{2} d \omega+(1-\sigma) \int_{\omega_{s}^{(k)}}^{\pi}\left|H_{0}^{(k)}\left(e^{j(\omega)}\right)\right|^{2} d \omega \\
& +(1-\sigma) \int_{0}^{\pi-\omega_{s}^{(k)}}\left|H_{1}^{(k)}\left(e^{j \omega}\right)\right|^{2} d \omega+(1-\sigma) \int_{s_{s}^{(k)}}^{\pi}\left(1-\left|H_{1}^{(k)}\left(e^{j \omega}\right)\right|\right)^{2} d \omega
\end{aligned}
$$

subject to (7),

where $\omega_{s}^{(k)}\left(\pi / 2<\omega_{s}^{(k)}<\pi\right)$ and $\pi-\omega_{s}^{(k)}$ are the stopband cut off frequencies of $H_{0}^{(k)}(z)$ and $H_{1}^{(k)}(z)$, respectively, $\sigma$ is a weighting constant from 0 to 1 , and $h$ is the vector containing the free variables in the impulse response. The transition bandwidth is $\Delta^{(k)}=2 \omega_{s}^{(k)}-\pi$. It is assumed that the FBs at stage $k$ are all identical. The constrained optimization is solved using the DCONF subroutine in the IMSL library. On average, it takes 150 iterations for convergence and the violation of $P R$ constraints is of the order of $10^{-15}$.

Given the number of channel $M=2^{K}$, transition bandwidth $\Delta^{(1)}$ and stopband attenuation $A$. The 2-channel PR LP FB at the first level is first designed by the above method to satisfy the given specification. Suppose that a filter length of $N^{(1)}$ is required. The $(K-1)$ 2-channel PR LP FBs at the other levels can be designed with parameters given in (5) and (6).

\section{B. DESIGN EXAMPLES}

We now present two examples: i) a two-level treestructured FB with $K=2$ and $M=4$, and ii) a three-level treestructured FB with $K=3$ and $M=8$. For simplicity, $N^{(1)}, N^{(2)}$. 
and $N^{(3)}$ are chosen as 128, 64 and 32 , respectively. The frequency responses of the three two-channel LP FBs are shown in Fig.3 (a), (b) and (c). It can be seen that they have approximately the same stopband attenuation but successively wider transition band. The frequency responses of the 4-band and 8-band LP analysis FBs obtained by cascading these 2-channel FBs in a tree structure are shown in Fig. 6 and Fig. 4, respectively. It can be seen that frequency characteristic of this LP 8-channel PR FB is very good and a stopband attenuation over $50 \mathrm{~dB}$ can be readily obtained. The design parameters of $H_{0}^{(k)}(z)$ are summarized in Table 1. As a final remark, we shall contrast the relative merits of this tree-structured $\mathrm{FB}$ and the LPPUFB. As mentioned earlier, the LPPUFB usually involves considerable number of parameters, especially when the number of channel and filter length is large. The objective function is also a highly nonlinear function of the planar rotation parameters. All these somewhat limits the stopband attenuation of the FB that can be designed. The proposed tree-structured FB is relatively easy to design because the 2-channel LP FBs can be designed separately. This limits the number of parameters in each sub problem to a reasonable value. Moreover, the design of 2channel LP PR FB is much easier than designing a LPPUFB and a number of efficient design methods are already available. On the other hand, the major disadvantage of the tree-structured FB is the restriction that the number of channel $M$ is a powers-of-two number. Though it is also possible to form tree-structured FB by cascading FBs with 2, 3 and larger number of channels using a similar approach, there is still a fundamental limitation on the number of channel that can be designed.

\section{CONCLUSION}

A method for designing $M$-channel LP PR FB with $M=2^{K}$ using a tree-structured FB is presented. It is based on a previous observation of Fliege, where the length of the analysis filters is decreased by a factor of two when the depth of the tree is increased by one, while its transition bandwidth is increased by the same factor. A lattice-based 2-channel LP FB is chosen because the frequency responses of the lowpass and highpass analysis (synthesis) filters can be designed to be closely symmetric to the other around $\pi / 2$. By properly selecting the filter length, transition bandwidth, and stopband attenuation of the 2-channel PR LP FBs at each stage of the tree structure, it is possible to design uniform PR LP FB with excellent frequency characteristic and much lower system delay.

\section{REFERENCES}

[1] N. J. Fliege, Multirate digital signal processing. John Wiley \& Sons Ltd, 1995.

[2] P. P. Vaidyanathan, Multirate systems and filter banks. Englewood Cliffs, NJ: Prentice Hall, c1992.

[3] R. D. Koilpillai and P. P. Vaidyanathan, "Cosine-modulated FIR filter banks satisfying perfect reconstruction," IEEE Trans. SP., Vol. 40, pp. 770-783, Apr. 1992.

[4] Y. P. Lin and P. P. Vaidyanathan, "Linear phase cosine modulated maximally decimated filter banks with perfect reconstruction," IEEE Trans. SP., Vol. 42, no.11, pp. 2525-2539, Nov. 1995.

[5] A. K. Soman, P. P. Vaidyanathan, and T. Q. Nquyen, "Linearphase paraunitary filter banks: Theory, factorizations and applications," IEEE Trans. SP., Vol. 41, pp. 3480-3496, Dec. 1993.

[6] T. D. Tran and T. Q. Nguyen, "On $M$-channel linear-phase FIR filter banks and application in image compression," IEEE Trans. SP., Vol. 45, pp. 2175-2187, Sept. 1997.

[7] R. L. de Queiroz, T. Q. Nguyen and K. R. Rao, "The GenLOT: Generalized linear-phase lapped orthogonal transform," IEEE Trans. on SP., Vol. 40, pp. 497-507, Mar. 1996.

[8] T. D. Tran, R. L. de Queiroz and T. Q. Nguyen, "Linear-phase perfect reconstruction filter bank: lattice structure, design, and application in image coding," IEEE Trans. on SP., Vol. 48, pp. 133 -147 , Jan. 2000.

[9] M. J. T. Smith and T. P. Barnwell III, "Exact reconstruction techniques for tree-structured sub-band coders," IEEE Trans. ASSP., pp. 434-441, Jume 1986.

[10] T. Q. Nguyen and P. P. Vaidyanathan, "Two-channel perfectreconstruction FIR QMF structures which yield linear-phase analysis and synthesis filters," IEEE Trans. SP., Vol. 37, pp. 676690, May 1989

[11] S. M. Phoong, C. W. Kim, P. P. Vaidyanathan and R. Ansari, "A new class of two-channel biorthogonal filter banks and wavelet bases," IEEE Trans. SP., Vol. 43, pp. 649-665, Mar. 1995.

[12] J. S. Mao, S. C. Chan and K. L. Ho "Desion of two-channel PR FIR filter banks with low system delay," Proc. IEEE ISCAS'2000, Vol. 1,pp. 627-630.

[13] K. Nayebi, T. P. Bamwell III and M. J. T. Smith, "Low delay FIR filter banks: design and evaluation," IEEE Trans. $S P$., Vol. 42, pp. 24-31, Jan. 1994.

[14] K. S. Pun, S. C. Chan, X. M. Xie, K. L. Ho and T. I. Yuk, "On the efficient realization and design of multiplier-less two-channel perfect reconstruction FIR filter banks," in student conference, IEEE ICASSP'2001

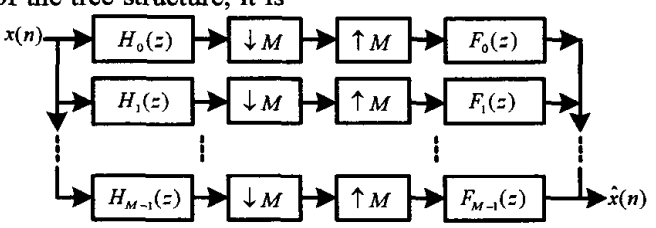

Fig. 1. The block diagram of a critically decimated uniform $M$-band FB.

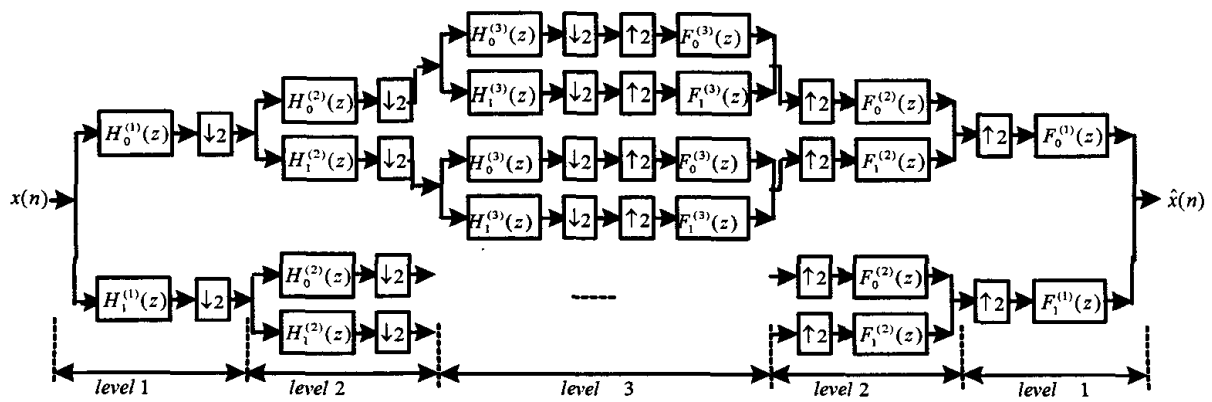

Fig. 2. 3-level maximally decimated tree-structured FB. 


\begin{tabular}{|c|c|c|c|}
\hline$H_{0}^{(k)}(z)$ for 4-band uniform FB & & $H_{0}^{(1)}(z), \quad k=1$ & $H_{0}^{(2)}(z), \quad k=2$ \\
\hline$H_{0}^{(k)}(z)$ for 8-band uniform FB & $H_{0}^{(1)}(z), \quad k=1$ & $H_{0}^{(2)}(z), \quad k=2$ & $H_{0}^{(3)}(z), \quad k=3$ \\
\hline Length of filter $N^{(k)}$ & 128 & 64 & 32 \\
\hline Stopband cut off frequency $\omega_{s}^{(k)}$ & $0.275 \times 2 \pi$ & $0.3 \times 2 \pi$ & $0.35 \times 2 \pi$ \\
\hline Transition bandwidth $\Delta^{(k)}$ & $0.025 \times 2 \pi$ & $0.05 \times 2 \pi$ & $0.1 \times 2 \pi$ \\
\hline Parameters to be optimized & 128 & 64 & 32 \\
\hline Implementation complexity & 33 MPUs & 17 MPUs & 9 MPUs \\
& 97 APUs & 49 APUs & 25 APUs \\
\hline
\end{tabular}

Table 1. Parameters of the lowpass filters in tree-structured FB.

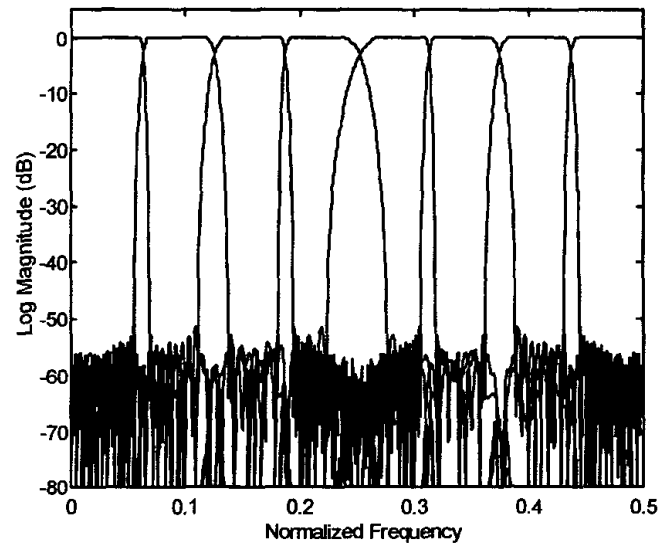

Fig. 3. The frequency responses of 3-level tree-structured FB by conventional method.

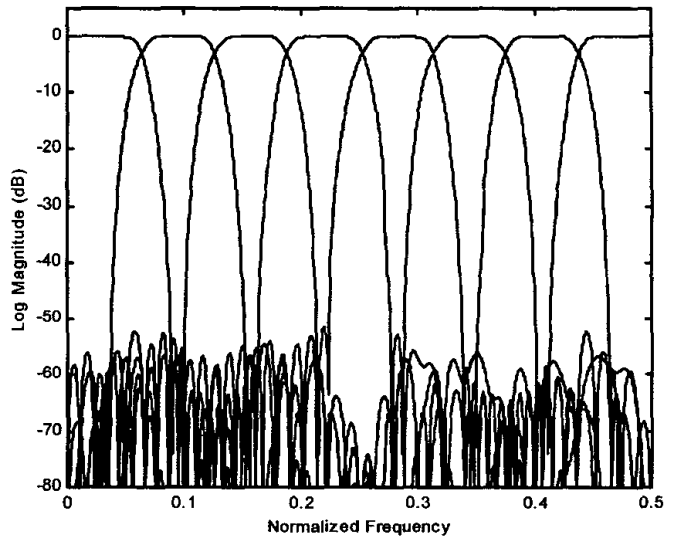

Fig. 4. The frequency responses of 3-level tree-structured FB by the proposed method.

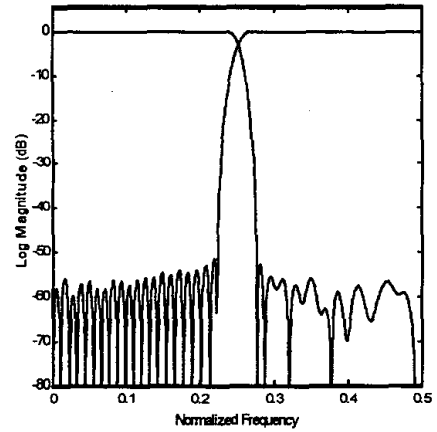

(a)

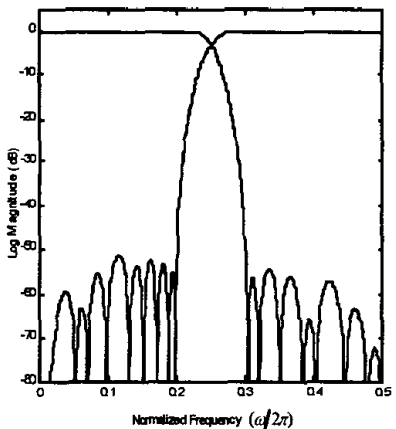

(b)

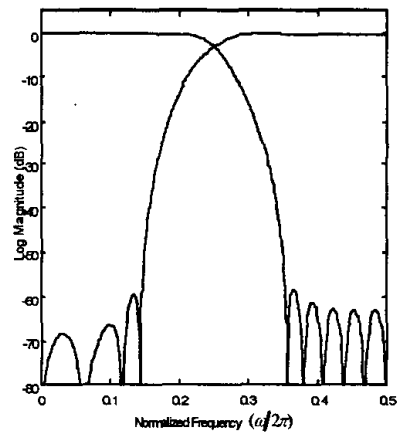

(c)

Fig. 5. Frequency responses of $\left\{H_{0}^{(k)}(z)\right.$ and $\left.H_{1}^{(k)}(z)\right\}$ in (a) level 1, (b) level 2, and (c) level 3.

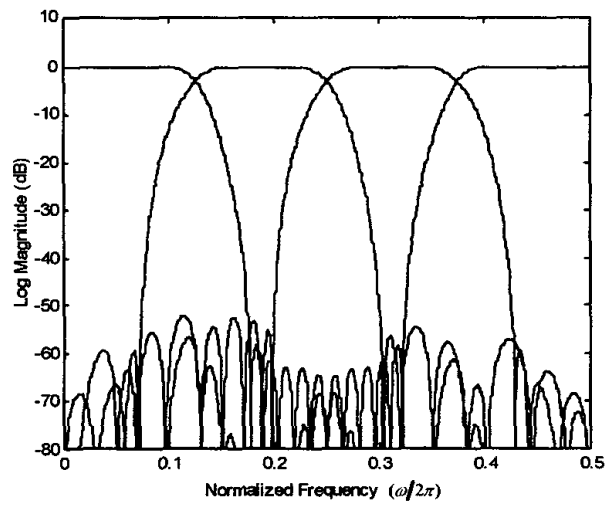

Fig. 6. The frequency responses of 4-band FB. 